https://doi.org/10.5719/aub-g/69.1/10

\title{
LA MIXITÉ SOCIALE, POUR UNE VIE COMMUNAUTAIRE A TRAVERS L'ESPACE PUBLIC. CAS DE AZZABA - ALGERIE
}

\author{
AMIRA AFRI ${ }^{1}$, BOUBA BENRACHI ${ }^{2}$
}

\begin{abstract}
Public space is a fundamental element in the development of cities and districts. It is viewed as a necessary mean for social interaction, ensuring a community life between inhabitants.

The public space that exists in Algerian cities is the most frequented and appreciated space by inhabitants, where different types of exchanges take place. However, this latter causes problems of structuring, inadequate planning and management, which prevent any social intensification. Indeed, reflections in matters of planning and management relating to the importance of this space as the keystone of the fabric of the city to live social mix and to urban qualification in the sense of landscape, are still absent in Algerian town planning instruments (Kettaf, 2015).

For it, this research is based on the study of inhabitant's perception of public spaces for the neighborhood Mefrouche Dahmène of Azzaba city, which is considered as a vector of social mix and living together promotion. This investigation methodology is based on using the mental map and the questionnaire, to understand what constraints are encountered in the Appropriate planning of public spaces and consequently in the evolution of social relations between inhabitants and the optimization of social diversity.

Keywords: Social diversity; public space; perception; planning; AZZABA.
\end{abstract}

\section{Introduction}

La vie sociale entre les habitants, ainsi que les relations qui s'y développent à travers les différentes activités qu'offrent la rue, le parking,

1 Doctorante, Laboratoire AUTES « De l'architecture à l'urbanisme, Espace, Techniques et société », Université Constantine 3 Algérie, amiraaf16@live.fr

2 Professeur, Laboratoire AUTES « De l'architecture à l'urbanisme, Espace, Techniques et société », Université Constantine 3 Algérie, bouba.benrachi@univ-constantine3.dz 
le trottoir, la place, l'espace vert, l'espace de jeu, le square, le jardin, l'équipement commerciale etc., s'accroissent toujours à travers les espaces publics. Ces espaces qui peuvent réunir autant de personnes pour se rencontrer, s'amuser, jouer ensemble, se balader, se reposer, etc., jouent un rôle crucial dans la vie des gens, en leur permettant de fuir pour un moment les problèmes du quotidien de la maison ou du travail. Rassemblant plusieurs personnes de différentes âges, sexes et catégories ou classes sociales, ces espaces optimisent le vivre ensemble par cette mixité sociale.

Le concept de mixité sociale ainsi favorisé par ces espaces, est une thématique qui constitue l'objet de multiples prises de positions et de débats au sein de la société, en faisant référence au brassage entre les habitants (Gerbeau, 2015: 1). Il apparaît comme une injonction exigée par des lois et des réglementations depuis plus de deux décennies dans certains pays de l'Europe (Dansereau, 2002: 23).

La réalisation de plusieurs types d'habitations dans une même assiette, réunissant logement individuel, collectif social et promotionnel et des équipements sociaux donne l'occasion de présence de plusieurs catégories sociales sur un même lieu de résidence, partageant ainsi les espaces publics proches. Par exemple en France la mixité sociale est prônée pour le vivre ensemble avec les améliorations apportées par la mise en œuvre de la loi d'égalité et de citoyenneté de 2016, favorisent l'égalité des chances dans l'habitat (Cosse, 2016: 1). En effet, Hélène Bacque et Fol mentionnent que «la mixité est affichée comme un objectif politique et a fait l'objet de plusieurs Lois à l'exemple de loi de solidarité et renouvellement urbain et de la Loi de l'orientation de la ville» (Bacque et Fol, 2008: 9).

Ce concept n'a pas de définition unique et simple. Dans le monde de la recherche on peut relever plusieurs approches relatives à la mixité sociale, (Hirtt et Delvaux, 2017 : 3), comme celles de Lelevrier (2008), Dallier (2010), et Hautière (2011), qui font de la mixité sociale un aspect propre à définir. Parmi les plus restrictives est celle de Lelevrier : " la mixité sociale est le moyen qui permet de développer un milieu de vie comme un lieu d'échange entre les habitants de différentes origines et différents niveaux de vie, en mettant en œuvre les conditions de vie et d'habitat qui favorisent la cohésion sociale entre les habitants avec un 
sentiment de sécurité et d'insertion dans leur espace de vie » (Lelevier, 2008: 8). En effet, bien avant c'est une expression d'action publique dont l'usage est relativement récent même si l'idée est ancienne, en particulier dans le champ des politiques de l'habitat et de la ville (Lelevrier, 2016: 33).

Cependant, ce concept de mixité sociale présente un champ d'investigation important quand on s'intéresse au développement urbain et à la vie au sein des quartiers (Baudin, 2001: 5). Il sert de base de réflexion pour l'élaboration et la mise en œuvre d'une approche globale pour la valorisation de zones d'habitation existantes (Kerivel \& James, 2019: 4). En effet, la mixité sociale est considérée comme un vecteur nécessaire à la vie qui permet le partage entre les citoyens, et limite le clivage social et les tensions (Belmessous, 2013 : 04). Son objectif est de vivre la ville ou le quartier dans un brassage entre les habitants, qui sont d'une diversité sociale avec différents caractéristiques sociaux, dans un même espace dit public (Belmessous, 2006: 4).

L'espace public est un concept important des sciences et des relations sociales (Tortel, 1998: 5). C'est un moyen nécessaire pour l'interaction sociale afin de s'ouvrir, de comprendre les autres, et entrer avec eux dans des conversations (Bourdin \& Cornier, 2017 : 8). Il est regardé comme le garant de la qualité de vie et de l'intégration sociale, et ainsi le ciment de la communauté (Voisin Bormuth, 2019: 1). En effet ce dernier représente le lieu de rencontre et de partage de la vie entre les habitants qui sont différents socialement pour y pratiquer différentes activités (jouer ensemble, vivre des moments de plaisir, se cultiver par les échanges, etc.)(Gros et Thibaud, 2001: 15). De même, la qualité de l'aménagement de cet espace est un enjeu décisif pour réussir cette mixité sociale (Voisin Bormuth, 2019: 3), car son organisation structure les relations sociales. Ces espaces représentent la vitrine du quartier ou de la ville et la scène de la vie urbaine (Monnet, 2012: 5). Fréquentés collectivement, ce sont des lieux d'usage, de communication et de circulation de divers points de vue des habitants qui résident ou non à proximité. (Dind, 2008: 2)

En effet, Moles et Rohmer expriment que «l'espace n'existe qu'à travers les perceptions que l'individu peut en avoir, qui conditionnent nécessairement toutes ses réactions ultérieures... » (Moles et Rohmer, 1972: 4). 
Donc, la perception de ces espaces représente les expériences vécues par l'individu qui l'utilise. Ce dernier construit en permanence des images des espaces dans lesquels il vit en s'appuyant sur l'évocation mentale du réel qui l'entoure. Dans ce cadre, la perception de l'espace n'est pas la réalité géographique, tangible, mais elle est nécessaire à son appréhension: c'est un acte cognitif, permettant d'accéder à la connaissance et à la compréhension voire à l'exploitation du monde. D'autre part, la perception s'appuie sur ce qui parait réel, l'extérieur et l'environnement, pour en forger une interprétation et en concevoir une image (Champagne, 2005: 8).

\section{Cas d'étude : le quartier Mefrouche Dahmène à AZZABA}

Azzaba est une des communes de la WILAYA de SKIKDA de l'Algérie, située dans son Sud-Est. Elle est d'une superficie de $206 \mathrm{Km}^{2}$ avec une population de 100.900 habitants et des capacités agricoles importantes. (www.wikepédia.com).

Le quartier Mefrouche Dahmène situé dans cette ville représente l'échelle choisie pour cette étude de recherche, il se localise dans la partie Est de la ville, d'une superficie de 17 ha, avec 408 logements (128 individuels et 280 collectifs). (Figures 1 \& 2)

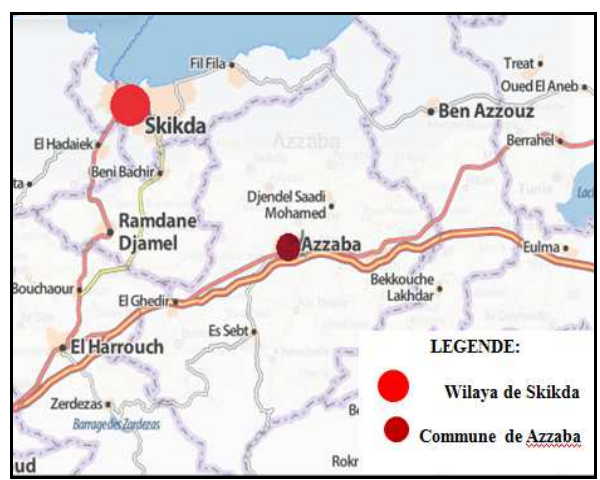

Fig.1. Situation de la ville d'Azzaba par rapport à la WILAYA

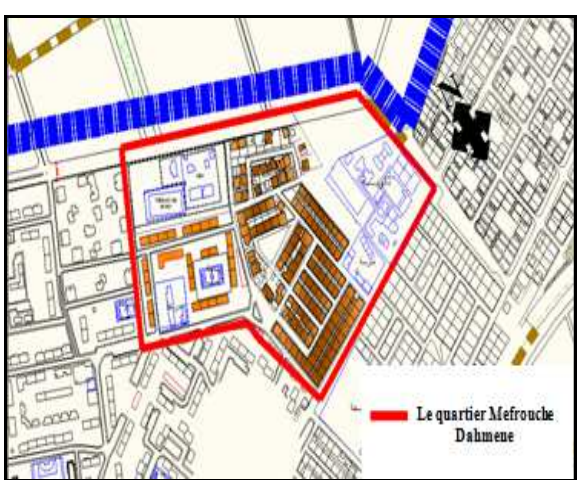

Fig. 2. Délimitation du quartier Mefrouche Dahmène de la ville d'Azzaba

Source : Plan directeur d'aménagement et d'urbanisme de la commune d'Azzaba, 2012, BEM 
Le choix a porté sur cette échelle, pour présenter un cas d'étude d'une petite ville algérienne ancienne, par ces habitants originaux, pour capter la problématique de mixité sociale et ainsi arriver à pencher l'étude d'impact de participation de l'espace public dans la favorisation de celle-ci.

\subsection{Méthodologie}

Dans cette recherche une étude méthodologique a été élaborée en se basant sur des outils de travail, ainsi que sur une analyse sociale et spatiale du quartier d'étude, la recherche met en exergue l'analyse du quartier par deux outils d'investigation : la carte mentale pour l'analyse de la perception spatiale, et l'enquête sur terrain à travers le questionnaire fermé pour l'analyse des compositions sociale et spatiale. Les résultats obtenus et le dépouillement du questionnaire ont été traités à l'aide de l'Excel.

Les cartes mentales représentent une méthode d'organisation des idées, qui sont utilisées pour capter la perception de l'espace public par les habitants sous forme de dessin et d'images mentales qui produisent des sensations en construisant un puissant lien entre l'individu et son milieu. Ainsi, ces cartes représentent les identifications données par les habitants à partir des lieux qu'ils fréquentent.

L'idée de la carte mentale a été d'abord introduite par les psychologues, dans la tentative d'étudier les modalités de la perception spatiale des individus. Elle a été successivement développée par les géographes comme outil d'analyse des espaces en tant qu'espaces perçus. Lynch (1960) a été parmi les premiers auteurs à employer les cartes mentales pour la représentation des espaces urbains. Cette construction de cartes mentales par les habitants du quartier nous mène à comprendre comment les gens perçoivent ces espaces publics à travers leur expérience d'usage. Cette perception s'appuie sur l'évocation mentale du réel qui l'entoure (Bailly, 1877: 4 ; Lynch, 1960: 6).

Pour élaborer ces cartes mentales, on demande aux personnes enquêtées de dessiner sur une zone de dessin dans la fiche d'enquête du questionnaire ce qu'ils considèrent comme espace public dans leur propre quartier, sans avoir la possibilité de regarder le paysage à 
représenter. Le but de ce dessin est de reproduire l'image mentale, filtrée, conceptualisée et mémorisée des lieux qui sont différents (rue, maison, place publique... etc.), sans que celle-ci soit influencée par des tentatives de se rapprocher de la réalité visuellement perçue par ces enquêtées. Bien sûr, les personnes interviewées sélectionnent certains éléments et en oublient d'autres. Des aspects sont soulignés, voire exagérés et d'autres minimisés.

En comparant les cartes dessinées entre elles, nous trouvons les déformations et les préférences en matière d'éléments retenus selon leurs profils sociodémographiques, leurs appartenances culturelles et leurs pratiques des lieux (éléments établis de manière complémentaire lors d'enquêtes). De ce fait, nous arrivons à démêler, comprendre, et expliquer la vision que les gens ont de ces espaces et les pratiques qui en découlent (Gould, 1974: 6).

L'utilisation avantageuse de la carte mentale comme outil de recherche pour étudier l'espace public, est de faire comprendre plus rapidement la situation de ces espaces, ainsi que d'établir des liens entre les idées des habitants; et connaitre quels sont les espaces publics les plus significatifs pour eux. Il est aussi à noter que certains inconvénients de ces cartes est la rencontre de cartes totalement vides et dont la question complémentaire à celle-ci est négative, ce qui ne correspond pas à notre objectif.

L'enquête qualitative, a été mise en place pour étudier notre sujet de recherche, par la planification d'un questionnaire complémentaire à la carte mentale, afin d'obtenir des réponses spontanées. Le questionnaire est destiné à normaliser et faciliter le recueil de témoignages des habitants, ou il est particulièrement investiguer des opinions, des attitudes, des croyances, des perceptions, des expériences ou encore des comportements. $\mathrm{D}^{\prime}$ autres informations concernant les personnes interviewées pourraient être connues et notées sans qu'il y ait besoin de poser des questionnes spécifiques. Cette méthode permet de toucher un plus grand nombre de personnes et atteindre une meilleure représentativité de la population étudiée.

L'objectif de cette enquête est de comprendre comment les habitants du quartier perçoivent leurs espaces publics, s'ils sont bien sécurisés, quel est l'usage et la fonction de ces espaces, et si le vivre ensemble à travers ces espaces publics existe entre eux. Dans son élaboration on 
s'est basé sur le contenu des deux concepts de notre étude : la mixité sociale et l'espace public, où trois indicateurs ont été retenus pour comprendre comment l'espace public favorise la diversité sociale à savoir ; la forme, le sentiment de sécurité et les fonctions et usages des espaces publics.

L'échantillonnage de celui-ci est composé de 102 habitants choisis sur un totale de 2040 habitants pour différentes caractéristiques à savoir : (âge, sexe, niveau culturel, profession, etc.). 38 femmes et 64 hommes interrogés avaient entre 18 et 70 ans (une tranche d'âge qui peut répondre aux questions de l'enquête), avec un nombre important ayant un âge entre 29 et 30 ans (une population jeune et plus sensible), et une large majorité de simples employés, chômeurs et retraités.

Ce questionnaire comporte 18 questions liées spécifiquement à nos concepts de recherche. Celui-ci a été testé sur cinq habitants avant sa distribution, pour vérifier sa compréhensibilité auprès des habitants, et calculer le temps que prennent les enquêtées pour remplir la fiche du questionnaire et qui estimée à $45 \mathrm{mn}$.

Le questionnaire commence par des consignes spécifiant toutes les caractéristiques des enquêtées (sexe, âge, professions, origine).

Selon nos indicateurs choisis pour cette étude, le questionnaire comprend cinq parties :

1. Relations entre les habitants du quartier: cette partie vise à comprendre comment les habitants du quartier partagent la vie ensemble (ex: Qui est le voisin pour vous? A quelle fréquence avez-vous les contacts avec les voisins ?)

2. La perception de l'espace public : elle comprend des questions ainsi des zones de dessins exprimées par la carte mentale qui doit montrer ce que l'habitant considère comme espace public dans son propre quartier, avec un complément de questions explicites par rapport à cette carte en cas de vide. (ex: Que Fréquentez-vous le plus dans votre quartier?

3. Le sentiment de sécurité dans l'espace public: pour comprendre si l'habitant est satisfait dans ces espaces, avec qui il se sent à l'aise... etc. 
4. Fonctions et usages des espaces publics : (ex: Pourquoi fréquentezvous les espaces publics de votre quartier? quel est la fonction de ces espaces ?)

5. Quelques questions complémentaires relatives aux attentes des habitants.

Les questionnaires ont été remis et repris en main propre de chaque enquêté. Ces opérations se sont déroulées entre le 08 mars et le 10 avril 2017, avec plusieurs passages pour pouvoir distribuer en main propre des habitants et le reprendre une fois complété.

Il est à remarquer que lors de l'analyse des données recueillies, 32 cartes mentales représentent un vide total sur l'identification visuelle de l'espace public. Sur ces 32 cartes mentales vides, 20 réponses ont été obtenues grâce à la question principale qui accompagne la carte mentale.

\subsection{Caractéristiques sociales et spatiales du quartier}

En ce qui concerne la composition sociale de la population enquêtée, le quartier dénombre plusieurs catégories sociales ce qui permet d'avoir une étude riche sur la notion de mixité sociale à travers l'espace public. Car les 2040 habitants du quartier sont différents dans leurs critères (socioprofessionnels, niveau de diplômes, l'origine, sexe, âge etc.)

Une dominance des habitants qui occupent les professions intermédiaires (les employés administratifs ainsi que les personnels de services) avec un pourcentage de $29 \%$, viennent ensuite les cadres avec $22 \%$ qui occupent des professions supérieures (directeurs de sociétés, d'entreprises... etc.), les ouvriers avec $19 \%$, les commerçants avec $13 \%$, les retraités avec $11 \%$, et enfin les chômeurs avec $6 \%$ de la population enquêtée (Figure 3). Ceci démontre une diversité socioprofessionnelle, qui encourage l'échange et le partage. 
LA MIXITÉ SOCIALE, POUR UNE VIE COMMUNAUTAIRE A TRAVERS L'ESPACE PUBLIC.

CAS DE AZZABA - ALGERIE

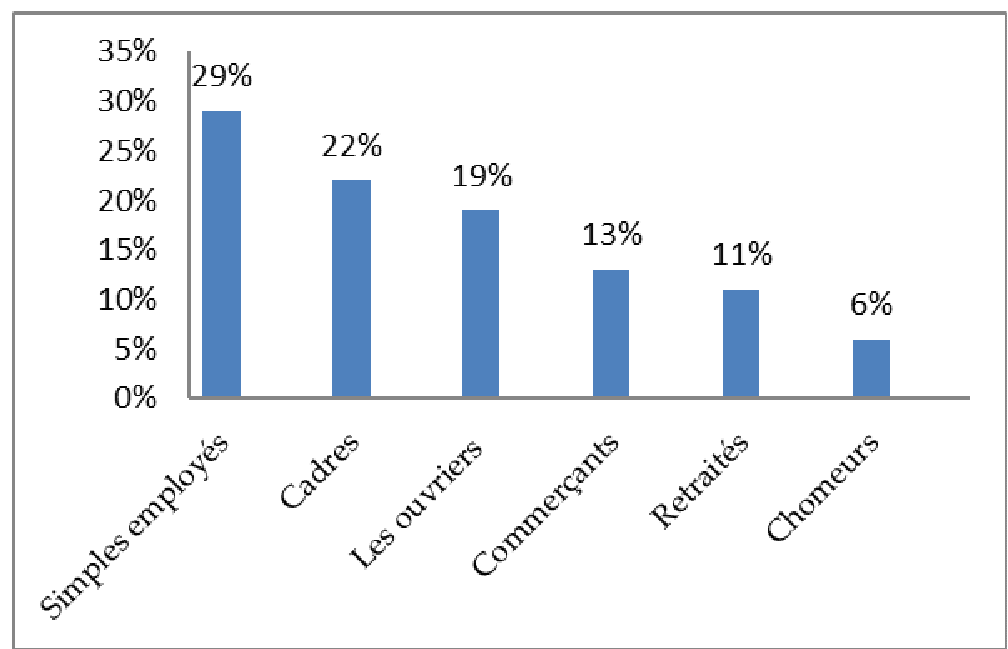

Fig.3. Le niveau socioprofessionnel des habitants du quartier Mefrouche Dahmène (en \%) Source : Auteur, 2017

On constate une diversité dans le niveau des diplômes et une grande représentativité des habitants avec un niveau supérieur de 32\%, suivi par ceux d'un niveau secondaire, et puis ceux des formations professionnelles, niveau moyen, et primaire respectivement avec $19 \%$, $14 \%$, et $9 \%$, et enfin les analphabètes avec $6 \%$ des habitants (Figure 4 ).

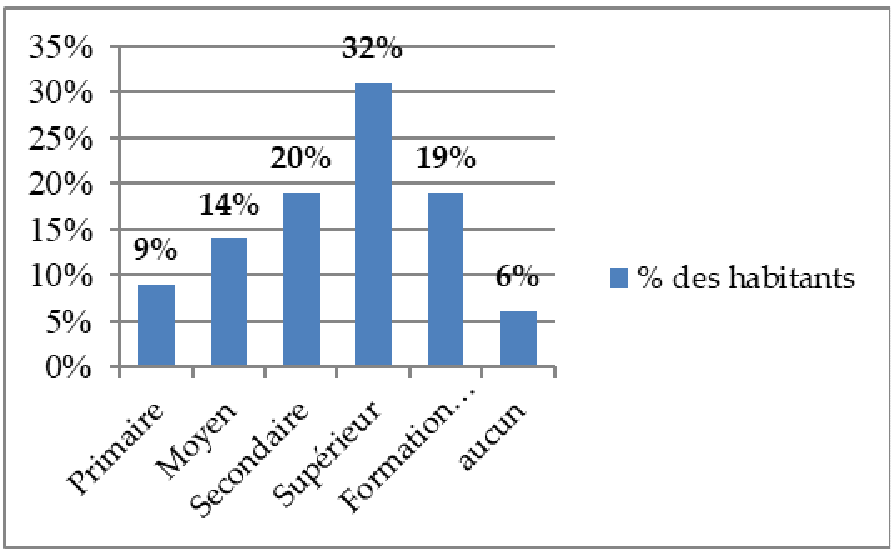

Fig. 4. Le niveau culturel des habitants du quartier Mefrouche Dahmène (en \%) Source: Auteur, 2017 
Le quartier comporte l'espace bâti composé d'habitat collectif, individuel, et les équipements, ainsi que l'espace non bâti qui compose les différents espaces publics (rues, espaces de jeux... etc.) (Figure5).

On distingue un nombre de 280 logements collectifs qui occupe une surface de 2.4 ha à savoir $14 \%$ de la surface totale du quartier, ainsi qu'un nombre de 128 logements individuels sur une surface de 4.9 ha, donc $29 \%$ de la surface du quartier.

Quant aux équipements, on distingue une école fondamentale située dans la partie nord du quartier, une cafétéria et des commerces de première nécessité implantés au rez-de-chaussée des bâtiments, deux écoles primaires, et un lycée, avec une surface totale de tous les équipements de 4 ha, c'est-à-dire $24 \%$ de la surface totale du quartier.

Il existe dans le quartier plusieurs types d'espaces non bâtis:

Les espaces de jeux : un terrain de jeux d'une surface de $2577 \mathrm{~m}^{2}$, et un terrain de sport d'une surface de $1262 \mathrm{~m}^{2}$, ainsi que des espaces marginalisés utilisés comme espaces de jeux par les enfants d'une surface de 1.2 ha. Tous ces derniers sont accessibles par toute la population du quartier.

Les voies de circulation: le quartier est bien desservi par une trame viaire importante, à travers la voie primaire située au nord, ainsi que par des voies secondaires et tertiaires intérieures.

Les parkings: des parkings sont implantés un peu partout dans le quartier.

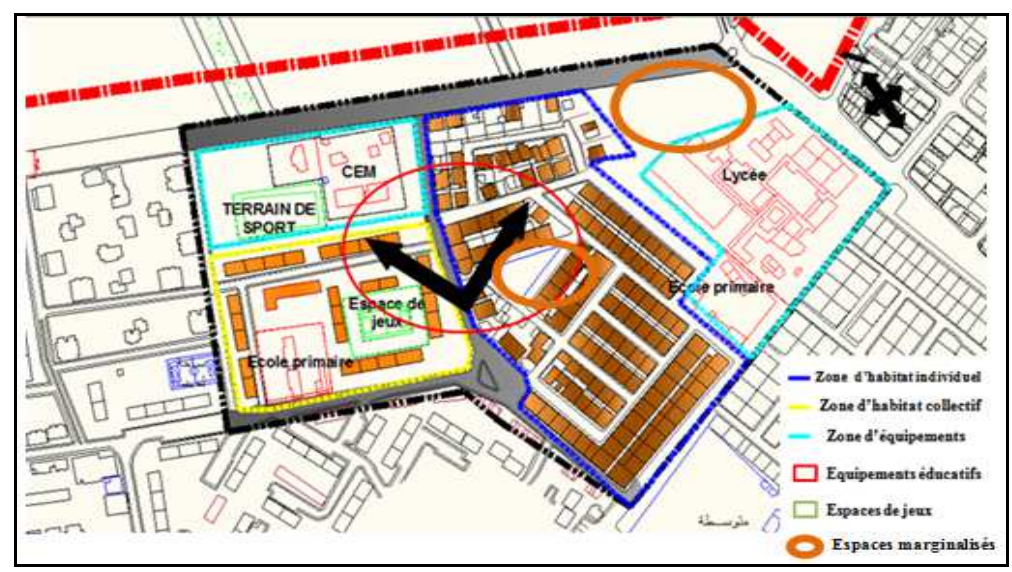

Fig. 5. Composition spatiale du quartier Mefrouche Dahmène Source: Traitement effectué par l'auteur du plan directeur d'aménagement et d'urbanisme de la commune d'Azzaba, 2012, BEM 


\section{La perception de l'espace public par les habitants pour vivre la mixité sociale : Résultat et discussions}

Selon l'étude des cartes ainsi que du questionnaire, la perception de l'espace public présentée par les habitants ne prend pas en considération tous les espaces qui constituent l'environnement. Elle présente seulement ceux qui sont les plus significatifs et intéressants pour eux. Par exemple : voirie et équipement, ou voirie, ou aires de jeux et voirie... etc. Les représentations des cartes sont différentes d'un individu à l'autre, puisque l'environnement, le bagage intellectuel, l'humeur du moment, le sexe, l'âge... etc., sont autant de paramètres qui conditionnent la perception que peut avoir un individu de son espace.

L'évaluation des trois indicateurs à savoir, la forme de l'espace public, le sentiment de sécurité et la fonction et l'usage de ce dernier, effectuée par la lecture et analyse des cartes mentales ainsi que du questionnaire a donné les résultats suivants :

\subsection{Une perception de l'espace public diversifiée}

L'espace public abordé par chaque individu et présenté dans la carte mentale de celui-ci montre un seul ou plusieurs espaces considérés comme espaces publics, malgré la présence de plusieurs types dans le quartier. Ce qui révèle la différence dans la vision des occupants par rapport à ces espaces, ainsi que dans la perception et la signification que $l^{\prime}$ habitant donne à ces espaces (Figures 6, 7, \& 8). 


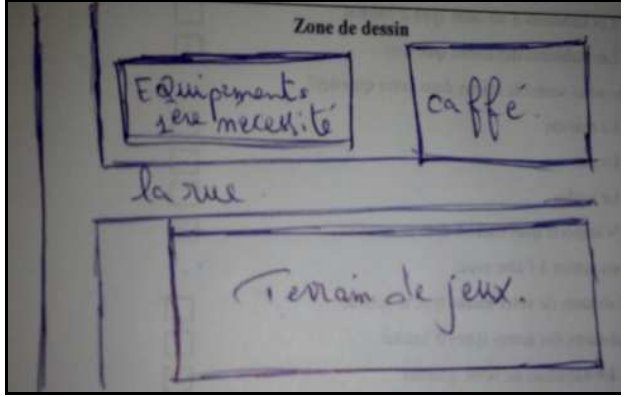

Fig. 6. Carte mentale de l'habitant A

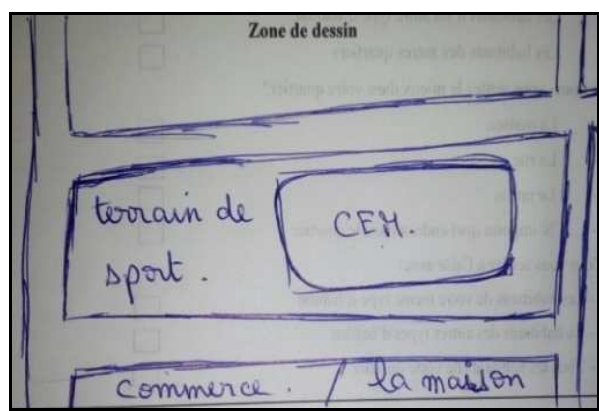

Fig. 7. Carte mentale del'habitant B

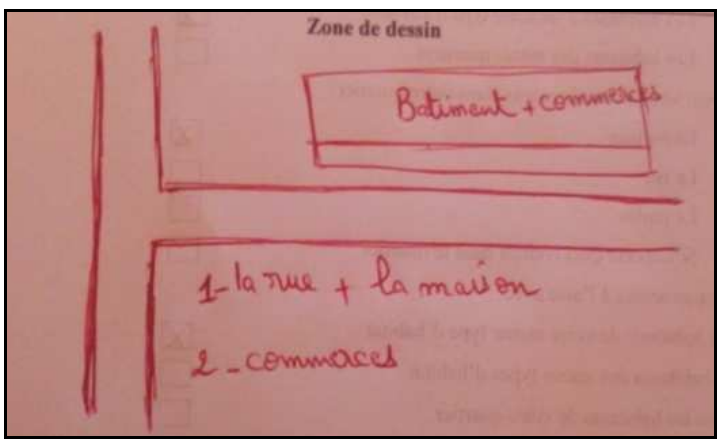

Fig. 8. Carte mentale de l'habitant C

Source : Auteur, 2017

Parmi les exemples des cartes présentées, la plupart indiquent les espaces les plus significatifs pour ces habitants dans leur quartier, quand d'autres montrent un vide total. Cependant, à travers les réponses complémentaires à ces cartes mentales données par le questionnaire sur la forme des espaces publics, on remarque que $44 \%$ des enquêtés font de la rue l'espace public le plus fréquenté, suivis par les aires de jeux avec $19 \%$, les équipements avec $14 \%$, et les lieux de travail avec $6 \%$. Quant aux $17 \%$ des habitants qui n'ont pas défini l'espace public de leur quartier dans la carte mentale et donc ils ont présenté cette dernière par un vide total, parce qu'ils utilisent ceux des quartiers environnants, parce qu'ils voient que ceux de leur quartier sont dans un état médiocre et non aménagé (Figure 9). 


\subsection{Un sentiment de sécurité dans l'espace public}

On observe que la majorité des habitants enquêtés se sente sécurisée dans son quartier, vu que ce dernier représente pour eux le lieu de partages de leur vie (Figure 12). Cependant certains paramètres importants favorisent ce sentiment, tels que les relations existantes entre les habitants. Car, l'enquête révèle qu'un échange de salutations et de conversations s'effectue toujours entre les habitants du quartier au niveau de la rue, des équipements et des espaces de jeux. En effet, les relations de voisinage entre les habitants, quel que soit le type d'habitat, sont différentes, car les échanges de salutations entre eux sont estimés à $47 \%$, les conversations représentent $26 \%$, ainsi que la visite des appartements ne présentent que 19\% (femmes). Tandis que $8 \%$ des habitants n'ont pas de contact entre eux (Figure 10). Il est à rappeler que l'ancienneté dans la ville d'Azzaba ainsi que l'origine des habitants restent toujours des points sérieux pour que les habitants restent fiers pour se partager des moments dans la vie d'ensemble.

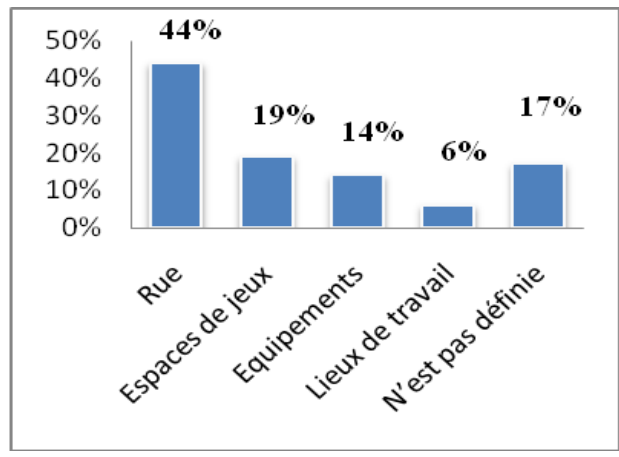

Fig. 9. Perception des espaces Publics selon la Forme (en \%)

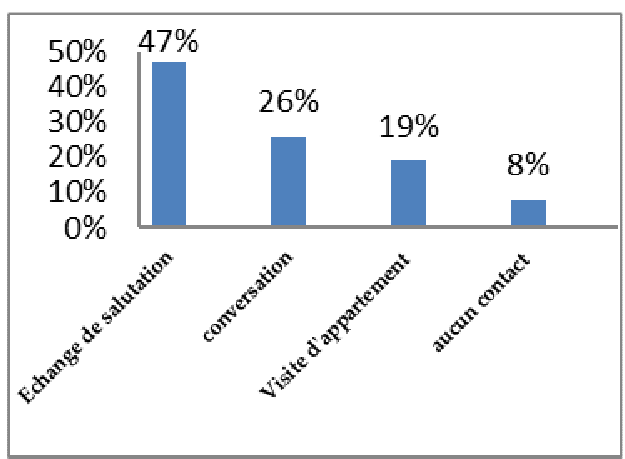

Fig. 10. Niveau de contact entre habitants dans le quartier (en \%)

Source : Auteur, 2017

\subsection{Fonction et usage de l'espace public}

La fréquentation de l'espace public par les habitants dans le quartier est différente. En effet, la majorité des habitants avec 52\% font de la rue 
l'espace le plus fréquenté, viennent après les espaces de jeux et la maison respectivement avec $41 \%$ et $37 \%$, et enfin on a les autres espaces, tels que ; les équipements et les lieux de travail avec 19\% et 3\% (Figure 11).

La fonction ainsi que l'usage de ces espaces sont différentes quel que soit sa typologie, la figure 12 montre que $55 \%$ des habitants enquêtés considèrent ces derniers comme des espaces de convivialité, viennent après $35 \%$ pour la détente et le loisir, $7 \%$ les fréquentent parce qu'ils sont les plus préférés pour eux et $3 \%$ pour autres.

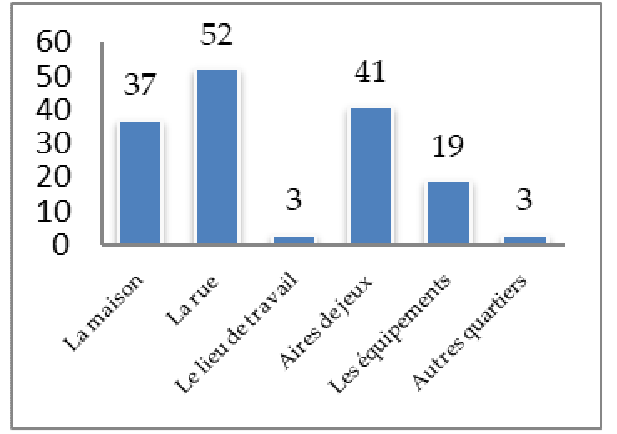

Fig. 11. Les espaces publics les plus fréquentés par les habitants du quartier (en \%)

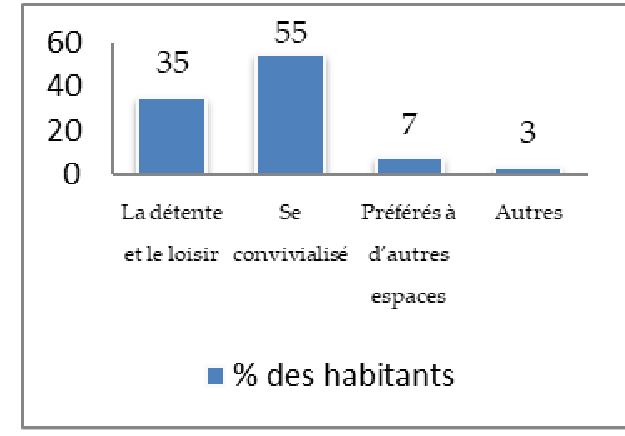

Fig. 12. Les différents usages de l'espace public (en \%)

Source : Auteur, 2017

Les résultats de cette recherche ont permis d'éclairer sur les différentes manières de perception de l'espace public par les habitants. Il en ressort aussi que la rue constitue l'espace public principal le plus fréquenté, ou se croisent le plus les habitants.

Ainsi il a été remarqué, quel que soit l'usage, la fonction ou les sentiments de sécurité, chaque habitant perçoit cet espace comme il l'entend dans son utilisation personnelle malgré le manquement dans son aménagement.

Toutefois, il est démontré que les aménagements adéquats de ces espaces sont nécessaires pour une fréquentation multiple et donc un renforcement des relations sociales et un vivre ensemble controverse avec un sentiment fort d'appartenance à ces espaces. Selon Monet «la qualité d'aménagement et l'organisation de l'espace public représentent le point qui structure les relations sociales » (Monet, 2012 : 5). 


\section{Conclusion}

La ville d'Azzaba, présente, selon l'étude, une vie commune active entre ses habitants qui sont natifs, où leur origine représente un point important pour le sentiment de sécurité. Ce dernier est aussi essentiel dans le développement de la mixité sociale. Cependant, la présence de ces éléments ainsi que de la diversité sociale dans ce quartier, à eux seuls sont insuffisants pour réussir la mixité sociale à travers ces espaces publics existants, malgré leur utilisation par les habitants. En effet, la présence de plusieurs contraintes à savoir ; la non-conformité à l'usage prédestiné, leur abondance par les collectivités locales et leur non affectation de fonctions ou aménagement, freinent le développement de cette mixité sociale. De plus, le concept de mixité sociale dans notre quartier en particulier ainsi que dans les structures urbaines de nos villes algériennes, est un concept qui n'est pas pris en considération dans les études préalables de la planification par les collectivités locales. Ce qui est à l'inverse de ce qui se passe dans les pays européens où celui-ci est toujours programmé et planifié, en amont comme l'exige la réglementation.

En conclusion, l'espace public est un lieu social et culturel, d'usage et de forme, et un élément important de création et de développement de la mixité sociale. Sa présence est de créer une vie sociale collective entre les habitants, quel que soit les caractéristiques sociaux (âge, sexe, niveau culturel, origine..., etc.). Dans notre cas d'étude, cette mixité existe d'une manière où la vie entre les habitants se partage à travers les espaces publics malgré la marginalisation dans leur aménagement. A cet effet, il est recommandé :

- Sur le plan local et à court terme une intervention direct sur ces espaces pour faire revivre la ville, que ce soit par le réaménagement et le renforcement d'usage de ces espaces, ou par la création des lieux conviviaux, où chacun peut exprimer ses propres besoins pour vivre ensemble.

- Sur le plan national et à moyen et long terme, une réflexion sur l'introduction du concept de mixité sociale dans la planification des quartiers ainsi que des villes à travers les outils d'urbanisme existants est à prendre en considération. 


\section{BIBLIOGRAPHIE}

Bailly, A.S., 1877, La perception de l'espace urbain : les concepts, les méthodes d'étude et leur utilisation dans la recherche géographique, thèse de doctorat d'état, Paris Sorbonne, $710 \mathrm{p}$.

Baudin, G., 2001, "La mixité sociale : une utopie urbaine et urbanistique », Les utopies de la ville, Revue du CREHU, $\mathrm{n}^{\circ} 10, \mathrm{pp} .13-23$.

Belmessous, H., 2006, Mixité sociale, une imposture : retour sur un mythe français, Collection comme un accordéon, Edition la Talande, 144p.

Belmessous, F., 2013, «Du seuil de tolérance à la Mixité sociale: répartition et mise à l'écart des immigrés dans l'agglomération Lyonnaise 1970-2000», Revue Belge de géographe, https://journals.openedition.org/belgeo/11540

Bourdin, S. \& Cornier, T., 2017, «Au-delà de la mesure du bien être en milieu urbain, quelle perception de la qualité de vie dans les villes européennes », Ecole de management de Normandie, UMR Idées 6266, in Géographie, Economie Société 19, pp. 3-31.

Brouand, J.P, 2015, «Droit de la cohésion territoriale, librairie générale de droit et de jurisprudence $», 230 \mathrm{p}$.

Cosse, E., 2016, Projet de loi Egalité et citoyenneté revient à la charge sur la mixité sociale, Université de Bourgogne, https://hal-univ-bourgogne.archives-ouvertes.fr/hal-01562581.

Dallier, F., 2010, Rénovation urbaine : La mixité sociale en différent, 4065.PHP.

Dansereau, F., 2002, La mixité sociale en habitation, Rapport de recherche réalisé pour le Service de l'habitation de la Ville de Montréal, Mai, INRS, Montréal.

Deschamps, E., 2005, Approche critique et juridique des normes relatives à la mixité sociale, dans le champ du logement, $N^{\circ} 125$. Pages 48 à 61, Caisse nationale d'allocations familiales, ISSN 0046-9459, https://doi.org/10.3917/inso.125.0048.

Dind, J., 2008, Principes de convivialité pour les espaces publics, les Cahiers de l'IAU, Paris.

Gerbeau, G., 2015, «Mixité sociale, la solution miracle qui cache les vrais enjeux », La Gazette des Communes, no 6/2256, 9 février, pp. 8-9.

Gibont, C., 2009, «L'espace public comme bien de transaction sociale, une lecture à partir des pratiques de loisir urbain » №20, revue Territoires, Villes, Environnement \& Société-EA 4477, université des Sciences et Technologies de Lille Avenue Paul Langevin 59655 Villeneuv-d'Ascq Cedex - France), pp. 153-165. https://hal.archives-ouvertes.fr/hal-01847996

Gros, J.M. \& Thibaud, J.P., 2001, L'espace urbain en méthodes, Ed. Parenthèses, Marseille, $224 \mathrm{p}$.

Hautiere, J., 2011, Mixité sociale, Travaux de recherche du PUCA.

Helene Bacque, M. et Fol, S., 2008, Les politiques de mixité sociale en France : de l'injonction politique nationale aux contradictions locales, Presses Sorbonne nouvelle.

Hirtt, N. \& Delvaux, B. (2017), « Peut-on concilier proximité et mixité sociale ? Simulation d'une procédure numérique d'affectation des élèves aux écoles primaires bruxelloises ", Les cahier de recherche du Girsef, 107, https://cdn.uclouvain.be/groups/cmseditors girsef/cahier_107_.Hirtt_Delvaux.Final.pdf.

Kerivel, A., \& James, S., 2019, Les enjeux de la mixité sociale en France, « fiches repères » INJEP (Institut national de la jeunesse et de l'éducation populaire), Mars, N 42. 
Kettaf, F., 2015, «La fabrique des espaces publics. Conceptions, formes et usages des places d'Oran (Algérie) », Les Cahiers d'EMAM [En ligne], 26 | 2015, mis en ligne le 16 juillet 2015. URL: http://journals.openedition.org/emam/995; DOI : https://doi .org/10.4000/emam.995

Lelevier, C., 2008, « Au nom de la mixité sociale ; les effets paradoxaux des politiques et rénovation urbaine, Savoir Agir, Editions du croquant, pp. 11-17.

Lelevier, C., 2016, La mixité sociale : sens et usages dans la politique de la ville, Regards sur la mixité sociale, Achevé d'imprimé en France par imprimerie de Champagne, 52200 Langres, Mars 2018.

Lynch, K., 1960, Carte mentale et présentations spatiales de résidents, 69002, Lyon.

Monnet, J., 2012, «Ville et loisir: les usages de l'espace public », Historiens EGéographes, $\mathrm{n}^{\circ} 419$, juillet-août, pp. 201-213.

Moles, A. \& Rohmer, E., 1972, Psychologie de l'espace, Paris, Casterman, 162 p.

PDAU, Plan Directeur d'aménagement et d'urbanisme de la commune d'Azzaba wilaya de Skikda, 2012, BEM.

Tortel, L., 1998, Une autre lecture de l'espace public : les apports de la psychologie de l'espace, Rapport d'études, CERTU.

Voisin Bormuth, C., 2019, Les espaces publics : clef du bien vivre ensemble, Ed la fabrique de la cité, Edito: Instant urbain, 24/06, https://www.lafabriquedelac ite.com/publications/les-espaces-publics-clef-du-bien-vivre-ensemble/ 
